\title{
POISSON STABILITY IN PRODUCT OF DYNAMICAL SYSTEMS
}

\section{A. KUMAR and R.P. BHAGAT}

Satgharwa, P.0. Buniad Ganj

GAYA (INDIA) Pin 823003

(Received July 10, 1986 and in revised form October 14, 1986)

ABSTRACT. This paper deals with Poisson stable and distal dynamical system. It is shown that the product of Poisson stable motions is a Poisson stable motion in dynamical systems.

KEY WORDS AND PHRASES. Distal dynamical system, compact, Poisson stable, recurrent motions.

1980 AMS SUBJECT CLASSIFICATION CODE. $34 \mathrm{C} 35$.

1. INTRODUCTION.

Product of dynamical systems is a dynamical system and product of semidynamical systems [1] is a semidynamical system. In [1] Bajaj has shown that if II $\alpha_{\alpha x_{a}}, \alpha \varepsilon I$ be the product of positive limit sets of the factor systems and $\Omega_{x}$ be the positive limit set of the product system then $\Omega_{x} \subset I I \Omega_{\alpha x_{a}}$. In general, c cannot be replaced by equality. Indeed $\Omega_{x}$ may be empty even if each of $\Omega_{\alpha x_{a}}, \alpha \in I$ is nonempty. He has found the conditions under which $\Omega_{1} x_{1} x_{2} x_{2}=\Omega_{x}$ and $\Omega_{x}$ could be nonempty and compact for a finite product.

We studied Poisson stable and distal dynamical systems in [2]. In this paper we shall mention the conditions under which $\Omega_{x}=I I \Omega_{\alpha x}, \alpha \varepsilon I$ and the product of Poisson stable motions is a Poisson stable motion in dynamical systems.

2. DEFINITIONS AND NOTATIONS.

We shall use the definition and notations of [2] and [3].

3. MAIN RESULTS.

PROPOSITION 3.1. If a motion $\pi(x, t)$ is Poisson stable and distal then its trajectory $\gamma(x)$ is a closed set. [2, theo.2.1].

THEOREM 3.2. If a compact motion is Poisson stable and distal then it is a compact recurrent motion.

PROOF. Let the motion $\pi(x, t)$ be Poisson stable and distal then its trajectory $\gamma(x)$ is closed hence $\gamma(x)=c 1 \quad \gamma(x)=\Omega_{x}$.

As the motion is compact each of the abovesets is compact and minimal, and thus by Birkhoff recurrence theorem $\pi(x, t)$ is compact recurrent.

THEOREM 3.3. Let $(X, \pi)$ be a semidynamical system, let $\pi$ be Lagrange stable, then $\pi$ is distal iff for every net $t_{i}$ in $R^{+}$, the phase space $X=\left\{z \varepsilon X: x t_{j} \rightarrow z\right.$ for some $x \in X$ and some subnet $t_{j}$ of $\left.t_{f}\right\}$. [4, theorem 2.6]. 
One can show that positive distal dynamical system are distal whenever all positive trajectory closures are compact. Thus the above result for semidynamical systems is applicable in present dynamical systems as we 11.

THEOREM 3.4. A compact motion $\pi(x, t)$ is Poisson stable and distal iff $\gamma(x)=\operatorname{cl\gamma }(x)=\Omega_{x}$ is compact and minimal.

PROOF. If a compact motion $\pi(x, t)$ is Poisson stable and distal then $\gamma(x)=\operatorname{cl\gamma }(x)=\Omega_{x}$ is compact and minimal.

Conversely, let $\gamma(x)=\operatorname{cl\gamma }(x)=\Omega_{x}$ be compact and minimal then it is compact recurrent and therefore compact Poisson stable.

To show the dynamical system $(X, \pi)$ is distal we sha11 use theorem 3.3. Here $Y(x)$ is compact and minimal, for every $x$ in $x$ the net $x t_{i}$ in $\gamma(x)$ has a subset $x t_{j} \rightarrow z$ in $r(x)$.

Thus $X=\left\{z \in X: x t_{j} \rightarrow z\right.$ for some net $t_{i}$ in $T$ and $t_{j}$ is some subnet of $t_{i}$ in $\left.\mathrm{T}\right\}$.

Which completes the proof.

THEOREM 3.5. The trajectory $\gamma(x)$ of a compact Poisson stable distal motion is complete and totally bounded.

PROOF. Since $\gamma(x)$ is compact in a Hausdorff uniform space $x$.

Now we shall consider product of such dynamical systems. Let $\left(\mathrm{X}_{\alpha}, \pi_{\alpha}\right), \alpha \in I$ be a family of dynamical systems. Let $X=I I X_{\alpha}$ be the product space. Let $x \in X, x=\left\{x_{\alpha}\right\}$. Define a map $\pi$ from $x x T$ into $x$ by $\pi(x, t)=\left\{x_{\alpha} t\right\}, \alpha \in I$ then $(X, \pi)$ is a dynamical system.

The dynamical system $(X, \pi)$ obtained above is called direct product or product of the family $\left(\mathrm{X}_{\alpha}, \pi_{\alpha}\right), \alpha \varepsilon \mathrm{I}$.

PROPOSITION 3.6. Let $\left(\mathrm{X}_{\alpha}, \pi_{\alpha}\right), \alpha \varepsilon I$ be a family of dynamical systems and $(\mathrm{X}, \pi)$ the product dynamical system. Let $x \in X, x=\left\{x_{\alpha}\right\}$. Then $\Omega_{x} \subset$ II $\Omega_{\alpha x \alpha}$.

THEOREM 3.7. Let $\left(\mathrm{x}_{\alpha}, \pi_{\alpha}\right), \alpha \in I$ be a family of \{Lagrange stable\} \{distal\} dynamical systems and $(\mathrm{X}, \pi)$ is the product dynamical system. Let $\mathrm{x} \varepsilon \mathrm{X}$ and $\mathrm{x}=\left\{\mathrm{x}_{\alpha}\right\}$ then $(\mathrm{X}, \pi)$ is \{Lagrange stable\} \{distal\}.

THEOREM 3.8. Let $\left(\mathrm{X}_{\alpha}, \pi_{\alpha}\right), \alpha \varepsilon \mathrm{I}$ be Lagrange stable distal dynamical systems. Let $\mathrm{x} \in \mathrm{X}, \mathrm{x}=\left\{\mathrm{x}_{\alpha}\right\}$ and $(\mathrm{X}, \pi)$ their product dynamical system, then the product $\pi(x, t)$ is Poisson stable motion in the product space $(X, \pi)$ iff each $\pi_{\alpha}\left(x_{\alpha}, t\right)$, $\alpha \in I$ is Poisson stable motion.

PROOF. We have $\gamma(x)=\operatorname{II} \gamma_{\alpha}\left(x_{\alpha}\right)=\operatorname{II~cl~} \gamma_{\alpha}\left(x_{\alpha}\right)=c 1 \operatorname{II} \gamma_{\alpha}\left(x_{\alpha}\right)=\operatorname{cl\gamma }(x)$. Also $\gamma(x)=\operatorname{II} \gamma_{\alpha}\left(x_{\alpha}\right)=I I \Omega_{\alpha x_{a}}=\Omega_{x}$. Therefore $\gamma(x)=\operatorname{cl} \gamma(x)=\Omega_{x}$, each being compact minimal and closed.

\section{REFERENCES}

1. BAJAJ, P.N. "Product of Semidynamical Systems", Recent Advances In Topological Dynamics, Springer Verlag lecture notes, No. 318, p. 23-29.

2. PRASAD, S.S. and KUMAR, A. "Stable P and Distal Dynamical Systems", Internat. J. Math. \& Math. Sc1. ? (1984), 181-185.

3. KUMAR, A. "Almost Periodic Motions in a Complete Space", Internat. J. Math. \& Math. Sci. 9 (1986), 621-622.

4. BHATIA, N.P. and NISHIHAMA, M. "Distal Semidynamical Systems", Dynamical Systems, An International Symposium, Vol. II, Edited by Cessari, Hale \& Lassale, A.P., 1976, p. 187-190. 


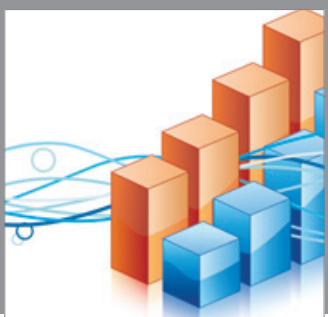

Advances in

Operations Research

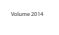

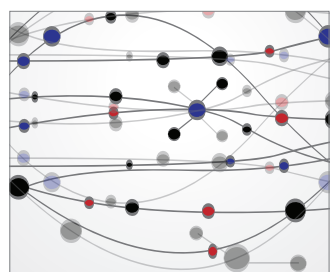

\section{The Scientific} World Journal
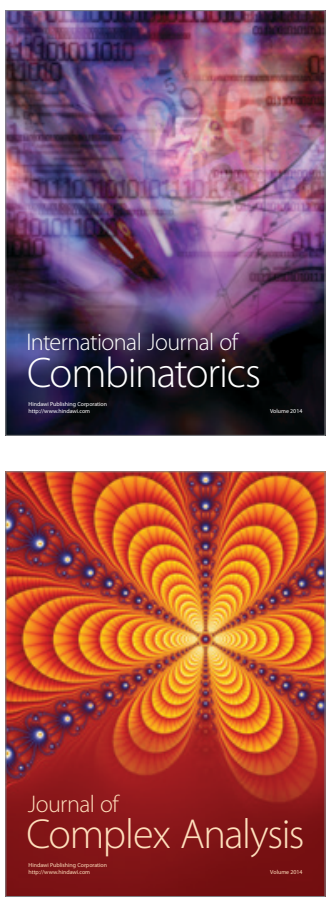

International Journal of

Mathematics and

Mathematical

Sciences
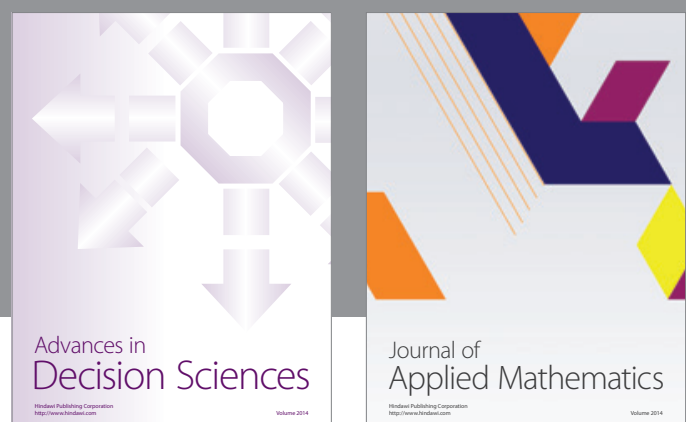

Journal of

Applied Mathematics
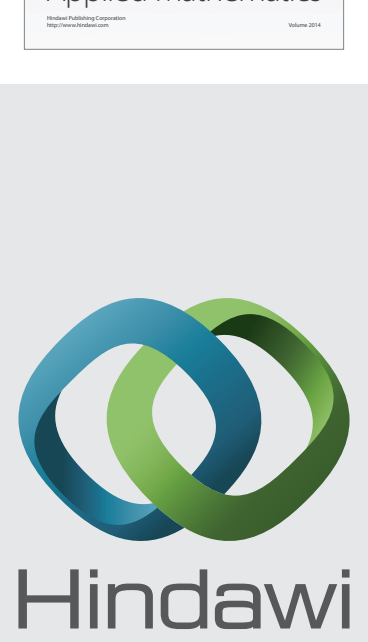

Submit your manuscripts at http://www.hindawi.com
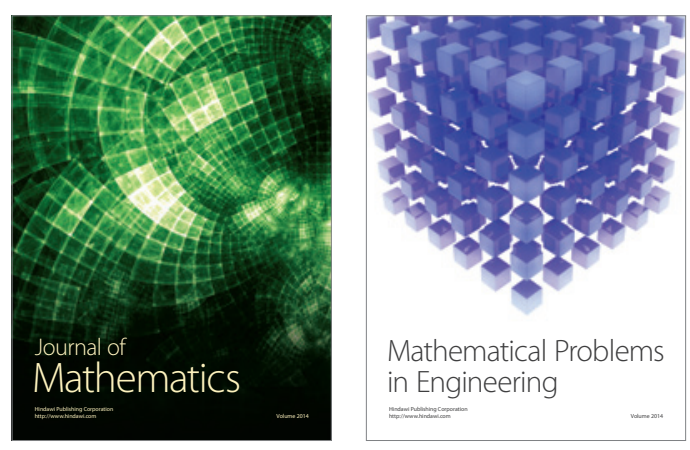

Mathematical Problems in Engineering
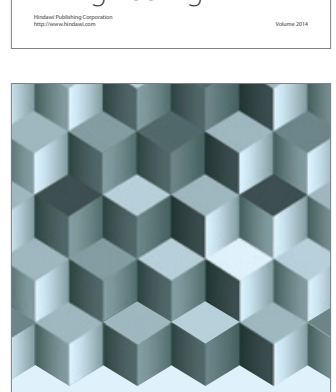

Journal of

Function Spaces
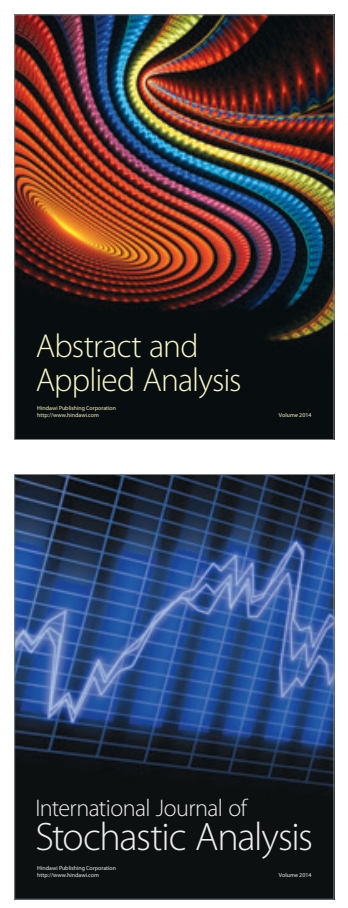

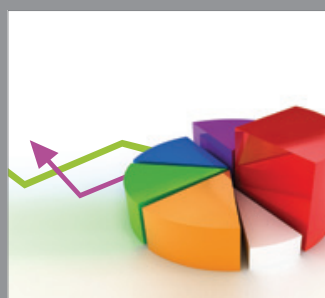

ournal of

Probability and Statistics

Promensencen
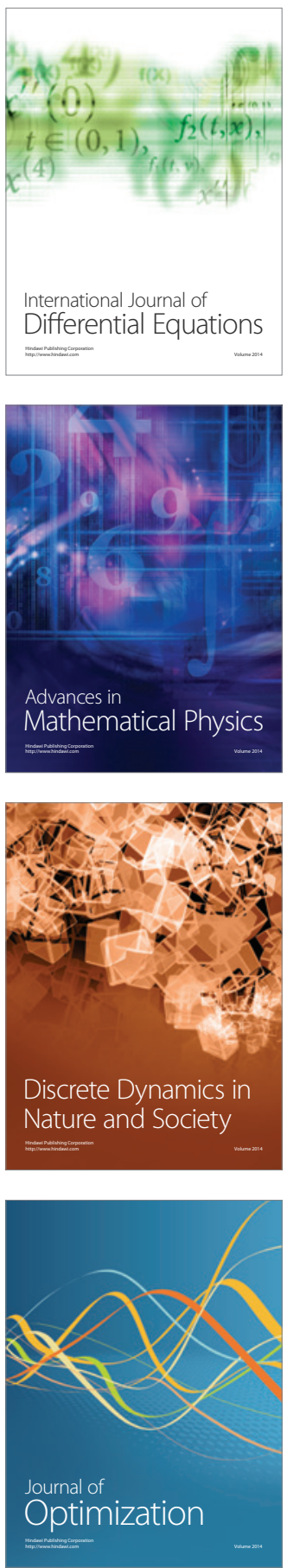